\title{
NUEVA GUÍA TÉCNICA PARA EL DISEÑO, FABRICACIÓN E INSTALACIÓN DE TUBERÍAS A PRESIÓN DE POLIÉSTER REFORZADO CON FIBRA DE VIDRIO
}

\author{
Berganza López, B. (1), Hernández Redondo, J.A(2), Andrés Martín, F.R. (3)
}

1) Jefe de Dep. Regadíos y Agua, TRAGSA, bberganz@tragsa.es

2) Jefe Oficina Operativa Extremadura, SEIASA, j.hernandez@seiasa.es

3) Laboratorio de Hidráulica, CEDEX, francisco.r.andres@cedex.es

\section{1) Resumen}

En los últimos dos años se ha estado trabajando en la elaboración de una nueva Guía Técnica para el diseño, fabricación e instalación de tuberías a presión de Poliéster Reforzado con Fibra de Vidrio. El objetivo perseguido ha sido recopilar la experiencia acumulada sobre este producto en las etapas anteriores en los distintos ámbitos o fases que abarca: diseño, fabricación e instalación. Con todos los actores implicados reunidos (fabricantes, usuarios, laboratorios, universidades y administraciones) se han revisado los procedimientos y normativas existentes para promover las máximas garantías de calidad y durabilidad. En concreto, se han incorporado novedades en cuanto a los estándares de calidad exigibles en la fabricación (nuevos ensayos de control de producto así como mayores prestaciones exigibles en las propiedades mecánicas), se ha revisado intensamente las diversas metodologías de cálculo mecánico existentes y se han comparado entre sí, y se han unificado criterios, como por ejemplo los de tipo dimensional, con el fin de simplificar la variabilidad vinculada a los diferentes procesos de fabricación. Por último, se ha tratado de estandarizar las recomendaciones sobre la instalación de las tuberías.

\section{2) Introducción. Objetivo del trabajo.}

En el sector del riego, la tubería de Poliéster Reforzado con Fibra de Vidrio es una tubería de amplia difusión. Resulta particularmente interesante la relación calidad/precio que ofrece, frente a otros tipos de materiales, el en el rango de diámetros DN500-1400 y presiones de trabajo PN 6- PN 16.

Hace dos años, aproximadamente, se formaron equipos de trabajo con fabricantes, usuarios, laboratorios, universidades y administraciones, con el ánimo de revisar la normativa existente y los códigos de buenas prácticas de instalación y mantenimiento de las tuberías de Poliéster Reforzado con Fibra de Vidrio. La experiencia adquirida en los últimos años con el uso de esta tubería permitía abordar el objetivo de elaborar un documento (Guía Técnica) en el que se unificaran criterios y se tratara de elevar el estándar de calidad (en la fabricación, montaje y explotación).

Actualmente existen en España tres métodos de fabricación de tuberías de PRFV, muy diferentes entre sí, (enrollamiento helicoidal, enrollamiento continuo y centrifugado) que le otorgan al producto características propias y particularidades que el usuario final no puede obviar. Por este motivo, se decidió tratar de consensuar y unificar criterios de diseño y de uso, con el fin de simplificar la citada variabilidad. 


\section{3) Descripción del proceso}

La Guía se ha estructurado en diferentes capítulos que se describen brevemente a continuación. En el presente documento se hará especial mención a las novedades incluidas en cada capítulo.

1) Consideraciones generales

Breve resumen de las definiciones de aplicación así como revisión de la normativa vigente. La Guía está especialmente dirigida a instalaciones enterradas a presión, para suministro de agua potable o de riego. Incluye especificaciones tanto para tubería como piezas especiales del sistema.

2) Componentes del sistema

Descripción de los elementos constituyentes del sistema: tubos, juntas y piezas especiales. Propiedades mecánicas y químicas exigibles, criterios de diseño y dimensiones.

En este apartado se ha tratado de unificar al máximo las diferencias dimensionales derivadas de los diferentes sistemas de fabricación y se ha prestado especial atención a nuevos requerimientos en cuanto a las propiedades mecánicas y químicas, a destacar la absorción de agua permitida por las resinas que se emplean en la fabricación de los tubos. Asímismo, se han incrementado los requerimientos de dureza Barcol a corto plazo como medida rápida en el control del nivel de curado de las resinas.

La Guía hace recomendaciones sobre las curvas de diseño de los tubos para la medida de la rigidez circunferencial a largo plazo. Se han incrementado los requerimientos de resistencia a la tracción circunferencial y longitudinal, respecto a los valores exigidos por norma. Respecto a las uniones, se recoge una descripción de los diferentes tipos de junta existentes en el mercado, con información técnica de tipo dimensional y mecánica. En cuanto a las piezas especiales, se ha tratado de ampliar información referente a criterios de diseño y constructivos, y acomodar a la nueva normativa los criterios de uso habituales.

3) Fabricación

Descripción y requerimientos de los materiales empleados en la fabricación de tubería y accesorios de PRFV (básicamente, resina de poliéster, fibra de vidrio y cargas inertes) y descripción de los principales sistemas de fabricación (enrollamiento continuo, enrollamiento helicoidal, centrifugado y laminación manual para piezas especiales y/o reparaciones-).

Especial mención requiere la introducción de nuevos criterios exigibles a las resinas (como es la absorción de agua admisible) y la recomendación de limitar el contenido de carbonato cálcico en la fabricación. Se unifican requerimientos sobre las materias primas, en cuanto a ensayos y propiedades, y se describe cada sistema de fabricación en el que se pone de manifiesto las particularidades propias de cada uno y la estructura tipo del laminado final, con especificación de las materias primas que se utilizan en cada capa estructural.

4) Diseño hidráulico

Breve descripción de los criterios habituales de diseño de las redes de riego y/o abastecimiento. Con especial atención a la definición de caudales en las redes y cálculo de las pérdidas de carga.

5) Diseño mecánico 
Comparativa entre los métodos habituales de cálculo mecánico para este tipo de tubería, AWWA M45 y CEN TR-1295-3 opción A. Se establecen recomendaciones en el uso de cada uno de los métodos al tener bases de cálculo diferentes y parámetros de instalación distintos, aún siendo ambos igualmente válidos. Así por ejemplo, el número de variables incluidas en el proceso de cálculo o la simplicidad en la elección de los parámetros que caracterizan los suelos, son algunas de las diferencias que aparecen entre cada uno de ellos.

6) Mantenimiento y reparación

Procedimiento para la ejecución de reparaciones en tubos y accesorios. Unificación de criterios respecto a las recomendaciones propias de cada fabricante. Especificaciones particulares para los diferentes sistemas de fabricación.

7) Consideraciones constructivas

Recomendaciones para la manipulación, transporte y almacenamiento de tubos y accesorios. Guía de instalación en zanja (con especial atención a los rellenos) y en condiciones especiales (tubería aérea o hinca). Anclajes, conexiones con obras de fábrica e instalación de accesorios (valvulería...).

En este apartado se han unificado los criterios y recomendaciones existentes y se han marcado los requerimientos mínimos de instalación, en particular, los relacionados con la calidad de los rellenos en zanja. La tubería de PRFV, por ser una tubería de tipo flexible, necesita de la contribución del terreno para resistir las cargas a las que se ve sometida, por esta razón, la instalación adquiere un papel relevante en el diseño, la comprobación y la ejecución de las secciones tipo de zanja correspondientes Se aportan, además, detalles constructivos para resolver la amplia variabilidad de elementos singulares que puedan aparecer en las redes (derivaciones, codos, valvulería, conexiones con arquetas...).

8) Gestión de la calidad

De especial relevancia en la Guía, se han revisado los ensayos prescritos por norma (para tubos y uniones, en la fabricación) y se han establecido nuevas frecuencias de control en fábrica. Del mismo modo, también se ha actualizado el procedimiento de prueba a la tubería instalada.Y en algunos casos se han incrementado los requerimientos de los ensayos normativos (se ha elevado el valor de dureza Barcol exigible a corto plazo y se han incrementado los requerimientos de tracción longitudinal y circunferencial por encima de los marcados por la norma de producto).

Por último, se ha incluido un Anexo con nuevos ensayos en proceso de definición y desarrollo, que buscan incrementar las exigencias de calidad al producto, sobre los ya requeridos por la normativa vigente. Estos ensayos hacen referencia a la dureza Barcol a corto y largo plazo, absorción de agua largo plazo de las resinas empleadas y del laminado, ensayo de impacto, ensayo de fatiga, ensayo de resistencia química a valores extremos de $\mathrm{pH}$ y deflexión a largo plazo en condiciones de $\mathrm{pH}$ ácido/ básico.

El ensayo de dureza Barcol a largo plazo pretende comprobar que la dureza de las resinas se mantiene por encima de unos valores mínimos durante la vida útil de la tubería, ya que se trata de una propiedad cuyo valor disminuye con el envejecimiento del material.

Se establecen los criterios básicos del ensayo, en fase de desarrollo, de absorción de agua de las resinas empleadas en la fabricación y del producto terminado (laminado) a largo plazo. Se pretende acotar los valores máximos exigibles dado que una de las causas de deterioro acelerado de este tipo de tubos se relaciona con la presencia de agua en su estructura interior.

El ensayo de impacto, de nueva inclusión, pretende medir la sensibilidad de los tubos a recibir golpes (la caída de piedras es un riesgo común en la instalación en zanja) y 
su capacidad a resistirlos sin sufrir daños estructurales que comprometan sus prestaciones mecánicas.

Con el ensayo de fatiga se pretende evaluar la pérdida de propiedades que sufren los tubos con el paso del tiempo (en concreto, su resistencia a soportar presión interna) cuando se ven sometidos a ciclos de presurización- despresurización (algo muy habitual en las redes de riego).

El ensayo de resistencia química a valores extremos de $\mathrm{pH}$ pretende garantizar la inercia química de las resinas empleadas frente a agentes externos que pudieran comprometer su viabilidad. Se busca reproducir el posible ataque al que se ven sometidas las conducciones en suelos agresivos, por hormigones o por presencia de fertilizantes disueltos en las aguas de transporte.

Por último, el ensayo de deflexión a largo plazo en condiciones extremas de $\mathrm{pH}$ pretende garantizar la resistencia a deflexión de los tubos a los largo de su vida útil. Este factor es de gran importancia en los diámetros grandes, ya que deflexiones por encima de las previstas pueden originar fugas indeseadas aún sin romper la tubería.

\section{4) Conclusiones}

El importante esfuerzo realizado por parte de la Sociedad Estatal SEIASA, la empresa pública TRAGSA así como el de todos los participantes en el Grupo de trabajo, ha dado como resultado una nueva Guía Técnica actualizada, con nuevos criterios y recomendaciones de diseño, fabricación e instalación unificados, y una propuesta de controles de calidad superiores a los requeridos por la normativa vigente. Se espera que la nueva Guía Técnica sea de referencia para facilitar el trabajo de los agentes involucrados: prescriptores, fabricantes, instaladores y usuarios finales. La intención que subyace, también, es garantizar unos estándares de calidad que redunden positivamente en las prestaciones ofrecidas por un producto de gran interés en el sector del riego.

\section{5) Agradecimientos}

Mención especial requiere en este apartado la labor realizada por el equipo de Luis Balairón desde el Centro de Estudios Hidrográficos del CEDEX, en su coordinación del grupo de trabajo y la elaboración de los documentos finales. En concreto, Francisco R. Andrés y Maria Isabel Berga. Cabe destacar también a otros colaboradores del CEDEX (Ángel Leiro, Ismael Carpintero y Jorge Mora). Nuestro agradecimiento a los fabricantes (Amitech, Protesa, Delta y Adequa), a los laboratorios (Instituto E. Torroja -Jose M. Chillón- y Silvia González, de la Universidad Rey Juan Carlos) y otros organismos colaboradores (ITACYL David Martínez-, ACUAMED y SARGA). Sin todos ellos no hubiera sido posible completar la elaboración de esta Guía Técnica. 